ARTIGO ORIGINAL/ORIGINAL ARTICLE

\title{
COMPOSIÇÃO CORPORAL DE PACIENTES COM DOENÇA RENAL CRÔNICA EM TRATAMENTO CONSERVADOR
}

\author{
BODY COMPOSITION IN CHRONIC KIDNEY DISEASE IN CONSERVATIVE \\ TREATMENT
}

DOI: $10.5380 /$ rmu.v1i2.40702

Daniela Barbieri Hauschild'; Maria Eliana Schieferdecker'; Christiane Mesquita Leite'; Marcelo Mazza do Nascimento

\section{RESUMO}

Introdução: A Doença Renal Crônica (DRC) caracteriza-se pela presença de lesão no parênquima renal e perda progressiva e irreversível das funções do rim. O estado nutricional é um importante preditor de prognóstico na DRC, inclusive na fase pré-diálise, o que ressalta a importância de estudos direcionados a avaliação da composição corporal desta população. Objetivo: Verificar a composição corporal em pacientes com DRC em tratamento conservador; bem como a associação da medida do ângulo de fase, com outros parâmetros nutricionais utilizados na prática clínica. Métodos: Estudo prospectivo observacional realizado no Ambulatório de Nefrologia do Hospital de Clínicas da UFPR, entre os meses de abril a julho do ano de 2011. A amostra foi composta de indivíduos com diagnóstico de doença renal crônica (DRC). Foi realizada avaliação nutricional por meio de antropometria, avaliação subjetiva global (ASG) e bioimpedância elétrica (BIA). Resultados: Foram avaliados 32 pacientes. Verificou-se correlação significativa e positiva entre o AF com a \%MLG e com IMME ( $r=0,4541$ e $p=0,009 ; r=0,5064$ e $p=0,003)$. O ângulo de fase foi significativamente menor em pacientes desnutridos classificados pela ASG $(p=0,01)$. Verificou-se uma correlação positiva e significativa entre CA e PCR ( $r=0,4053$ e $p=0,0262$ ). Conclusão: Nesta casuística, os dados antropométricos indicaram sobrepeso, obesidade e acúmulo de gordura corporal. O AF parece identificar alterações de composição corporal que antecedem alterações de parâmetros antropométricos. Pode-se verificar que a ASG pode ser uma ferramenta adequada na avaliação nutricional desta população.

Palavras-Chaves: Insuficiência Renal Crônica; Desnutrição; Avaliação Nutricional; Impedância elétrica.

\section{ABSTRACT}

Introduction: Chronic Kidney Disease (CKD) is characterized by the presence of lesions in the renal parenchyma and progressive and irreversible loss of kidney function. Nutritional status is an important predictor of prognosis in CKD, including predialysis phase, which underscores the importance of studies addressing the assessment of body composition in this population. Aim: To assess the body composition in CKD patients, and the association between phase angle and other nutritional parameters used in clinical practice. Methods: A prospective observational study conducted at the Clinic of Nephrology of the Federal University of Parana, between April and July of 2011. It included patients with CKD and the nutritional status was assessed by anthropometry, subjective global assessment (SGA) and bioelectrical impedance analysis. Results: A total of 32 patients were evaluated. There was significant and positive correlation between the phase angle and skeletal mass index $(r=0.4541$ e $p=0.009 ; r=0,5064$ e $p=0,003)$. The phase angle was significantly lower in malnourished patients classified by $S G A(p=0,01)$. There was a positive and significant correlation between the waist circumference and the C-reactive protein ( $r=0.4053$ e $p=0.0262)$. Conclusion: in this study, it was found, according to anthropometric measurements, patients with overweight, obesity and high body fat. The phase angle seems to identify body composition changes that precede changes of the anthropometric parameters. The SGA is a good tool for nutritional assessment in this population.

Key words: Chronic Kidney disease. Malnutrition. Nutrition assessment. Electric impedance.

1-Universidade Federal do Paraná

Contato do Autor / Mail to:

Daniela Barbieri Hauschild - danielahauschild@yahoo.com.br

Manoel Ribas, 8501, casa 178, Santa Felicidade, Curitiba, Paraná CEP:82400-000 


\section{INTRODUÇÃO}

A doença renal crônica (DRC) constitui-se em um problema de proporções mundiais. No Brasil, estima-se que aproximadamente 131.000 pessoas estejam nos estágios iniciais da $\mathrm{DRC}^{1,2}$. A desnutrição energético-protéica frequentemente está presente nos pacientes com DRC em tratamento conservador, decorrente, principalmente, do processo inflamatório crônico3. Porém, a maior parte dos estudos a respeito do estado nutricional em pacientes renais crônicos ocorre em situações de hemodiálise ou diálise peritoneal ${ }^{4}$.

Na avaliação do estado nutricional, na DRC, são utilizados diversos parâmetros, como medidas antropométricas, exames laboratoriais e bioimpedância5. A bioimpedância elétrica é uma técnica objetiva, simples e não invasiva utilizada para estimar a composição corporal. É um método que consiste na passagem de uma corrente elétrica pelo corpo, permitindo mensurar a resistência e a reactância dos compartimentos corporais ${ }^{6,7}$. Parte da corrente elétrica é armazenada pelas membranas celulares, as quais agem como verdadeiros capacitores, no que resulta em uma mudança de fase elétrica, quantificada geometricamente, por meio da impedância, como ângulo de fase (AF). Baixos valores de $A F$ refletem aumento da água intra e extracelular e redução da massa celular corporal. Como conseqüência, o ângulo de fase tem sido utilizado como indicador prognóstico independente de sobrevida em diversas enfermidades, entre elas, a DRC $^{4,8,9,10,11}$.

Por outro lado, a obesidade tem atingido proporções endêmicas, refletindo-se também nos doentes renais crônicos ${ }^{12}$. Estudos apontam que elevado índice de massa corporal (IMC) teria efeito protetor no prognóstico da doença. No entanto, acúmulo de gordura não pode ser avaliado de forma isolada, pois mesmo com IMC elevado, os pacientes podem apresentar desnutrição protéica pela avaliação subjetiva global (ASG) e baixos valores de massa livre de gordura, conhecida como sarcopenia do obeso $^{12,13,14}$.

O objetivo do estudo foi verificar a composição corporal em pacientes com DRC em tratamento conservador; bem como a associação da medida do ângulo de fase, com outros parâmetros nutricionais utilizados na prática clínica.

\section{METODOLOGIA}

Estudo prospectivo observacional realizado no Centro de Pesquisas Nefrológicas do Hospital de Clínicas da Universidade Federal do Paraná (HC-UFPR), entre os meses de abril a julho do ano de 2011. A amostra foi composta de indivíduos com diagnóstico de doença renal crônica (DRC), de acordo com ritmos de filtração glomerular estimado por meio da fórmula proposta pela National Kidney Foundation, $2002^{15}$. Foram excluídos aqueles pacientes com presença de câncer ou doença hepática, estado inflamatório agudo e/ou idade superior a 80 anos ou inferior a 18 anos.

Os pacientes foram submetidos à avaliação nutricional, no período da manhã, em jejum de 10 a 12 horas. A avaliação foi composta de indicadores clínicos, antropométricos, bioquímicos e impedância elétrica.

Como indicador clínico foi realizada a avaliação subjetiva global (ASG) adaptada de Detsky et al $(1987)^{16}$ e Detsky, Smalley e Chang $(1994)^{17}$. Os dados antropométricos incluíram peso, estatura, circunferência do braço $(\mathrm{CB})$, circunferência abdominal (CA) e prega cutânea triciptal (PCT). Posteriormente foram calculados os dados de circunferência muscular do braço (CMB) e índice de massa corporal (IMC). Para classificação do IMC, foi utilizada a recomendação de pontos de corte para adultos estabelecida pela Organização Mundial de Saúde $(1998)^{18}$ e para a população idosa a proposta de Lipschitz $(1994)^{19}$. O cálculo da adequação, os valores de CB e PCT foram comparados com referencial de Frisancho $(1981)^{20}$, e os valores de CMB com Frisancho (1990) ${ }^{21}$. Para idosos, foi utilizado o referencial de Burr, ML e Phillips $(1984)^{22}$. Estes parâmetros foram classificados de acordo com Blackburn e Thorton (1979) ${ }^{23}$. Para classificação de risco cardiovascular, foram utilizados os pontos de corte de CA propostos pela Organização Mundial de Saúde: sem risco cardiovascular, risco cardiovascular e muito risco cardiovascular ${ }^{24}$.

A avaliação bioquímica incluiu dados séricos de albumina, por meio do método verde de bromocresol; creatinina, pelo método picrato alcalino; e proteína C reativa (PCR) ultrassensível, por imunonefelometria, realizados pelo serviço de bioquimica do Hospital de Clínica - UFPR.

A impedância elétrica foi realizada com a utilização do aparelho de análise de composição corporal tetrapolar RJL Systems ${ }^{\circledR}$ modelo Quantum BIA 101Q Clinton Township, Michigan, Estados Unidos, para a obtenção do percentual de massa livre de gordura (\%MLG) do percentual de gordura corporal (\%GC) e, o Índice de massa esquelética (IMME). Para a obtenção dos valores de \%MLG e \%GC, foi utilizado o software Body Composition Assessment (RJL Systems ${ }^{\circledR}$ ), Champaign, Illinois, Estados Unidos, 1997. Os valores de ângulo de fase (AF) foram calculados por meio da fórmula: $A F=$ reactância/resistência $x$ $180^{\circ} / 3,14$. O IMME foi calculado por meio da equação ${ }^{26}$ IMME=MLG $(\mathrm{kg}) /$ Altura $(\mathrm{m})^{2}$.

Para a análise estatística foi utilizado o software R version 2.11.1 (2010-5-31). Os resultados descritivos foram apresentados em frequência, média e desvio padrão. De acordo com a distribuição gaussiana das variáveis, foi utilizada para correlação 
entre as variáveis o Coeficiente de Pearson e os testes de Mann-Whitney e Kruskal-Wallis. Adotou-se o nível de significância de $5 \%(p<0,05)$.

O estudo foi aprovado pelo Comitê de Ética em Pesquisa em Seres Humanos do HC-UFPR (protocolo 2412.019/2011-02)

\section{RESULTADOS}

\section{Parâmetros clínicos e antropométricos}

Foram avaliados 32 pacientes, sendo $62,5 \%$ do sexo masculino com idade média de 58,3 anos, a maior parte eram idosos e todos os pacientes eram hipertensos. As características clínicas e laboratoriais dos pacientes encontram-se na Tabela 1.

Aproximadamente $15 \%$ dos pacientes apresentaram desnutrição protéica-energético de acordo com a ASG, $3,1 \%$ pelo IMC, $15,9 \%$ pelo $C B$ e $12,9 \%$ pela $C M B$. No entanto, pelo IMC, CA e PCT, a maior parte da população encontrava-se na faixa do sobrepeso/obesidade (65,6\%; $75,0 \% ; \quad 51,6 \%$ respectivamente).

\section{Bioimpedância e ângulo de fase}

Verificou-se correlação significativa e positiva entre o AF com a \%MLG e com IMME ( $r=0,4541$ e $p=0,009 ; r=0,5064$ e $p=0,003$, respectivamente) (Figura $1 \mathrm{~A}$ e B). Por outro lado, não houve associação entre $A F$ com IMC ( $r=0,17$ e $p=0,3521)$; CA $(r=0,0753$ e $p=0,6821) ; \% C B(r=0,0874$ e $p=0,634) ; \% P C T(r=02942$, e $p=0,1081) ; \% C M B(r=-0,0363$ e $p=0,8462)$; albumina $(r=0,1988$ e $p=0,2921)$; PCR $(r=-0,0278$ e $p=0,8839)$; e ritmo de filtração glomerular estimado (RFGe) ( $r=-$ $0,1261$ e $p=0,4916)$.

Quando os pacientes foram classificados em desnutrição e eutrofia, de acordo com a ASG, \%CB, $\% \mathrm{PCT}$ e \%CMB, verificou-se que o ângulo de fase foi significativamente menor apenas em pacientes desnutridos classificados pela ASG (5,62 versus 6,88; $p=0,01$ ).

\section{Circunferência Abdominal}

Na classificação de acordo com a CA, em sem risco, risco e alto risco cardiovascular, verificou-se que as médias de IMC, \%CB e \%GC foram inferiores nos indivíduos considerados em eutrofia, quando comparados a pacientes com elevado risco cardiovascular. Não houve diferença entre os grupos com relação ao IMME (Tabela 2).

Como era de esperar verificou-se uma correlação positiva e significativa entre CA e PCR $(r=0,4053$ e $p=0,0262$ ) (Figura 1C).
Tabela 1. Caracterização dos pacientes portadores de doença renal crônica

\begin{tabular}{|c|c|c|c|}
\hline & Feminino & Masculino & Total \\
\hline$n(\%)$ & $12(37,5)$ & $20(62,5)$ & 32 \\
\hline Idade (anos) & $55,28( \pm 14,7)$ & $\begin{array}{c}60,25 \\
( \pm 10,3)\end{array}$ & $\begin{array}{c}58,30 \\
( \pm 12,19)\end{array}$ \\
\hline Hipertensão (\%) & 100 & 100 & 100 \\
\hline $\begin{array}{l}\text { Diabetes mellitus } \\
\text { (\%) }\end{array}$ & 33,3 & 25 & 28 \\
\hline IMC $\left(\mathrm{kg} / \mathrm{m}^{2}\right)$ & $28,72( \pm 6,48)$ & $\begin{array}{c}27,68 \\
( \pm 4,07)\end{array}$ & $\begin{array}{c}28,07 \\
( \pm 5,03)\end{array}$ \\
\hline $\mathrm{CA}(\mathrm{cm})$ & $\begin{array}{c}98,03 \\
( \pm 14,06)\end{array}$ & $\begin{array}{c}99,74 \\
( \pm 12,89)\end{array}$ & $\begin{array}{c}99,10 \\
( \pm 13,14)\end{array}$ \\
\hline CB (\%) & $\begin{array}{l}108,07 \\
( \pm 15,06)\end{array}$ & $\begin{array}{c}102,44 \\
( \pm 16,87)\end{array}$ & $\begin{array}{l}104,55 \\
( \pm 16,2)\end{array}$ \\
\hline РCT (\%) & $\begin{array}{c}85,16 \\
( \pm 39,35)\end{array}$ & $\begin{array}{c}123,62 \\
( \pm 49,84)\end{array}$ & $\begin{array}{c}109,97 \\
( \pm 49,39)\end{array}$ \\
\hline CMB (\%) & $\begin{array}{l}113,36 \\
( \pm 9,43)\end{array}$ & $\begin{array}{l}103,45 \\
( \pm 15,5)\end{array}$ & $\begin{array}{l}106,96 \\
( \pm 14,32)\end{array}$ \\
\hline Ângulo de fase & $6,24( \pm 1,1)$ & $6,94( \pm 1,49)$ & $\begin{array}{c}6,68 \\
( \pm 1,38)\end{array}$ \\
\hline MLG (\%) & $60,99( \pm 6,02)$ & $\begin{array}{c}72,06 \\
( \pm 7,52)\end{array}$ & $\begin{array}{c}67,91 \\
( \pm 8,78)\end{array}$ \\
\hline GC (\%) & $39,01( \pm 6,02)$ & $\begin{array}{l}27,95 \\
( \pm 7,52)\end{array}$ & $\begin{array}{c}32,09 \\
( \pm 8,78)\end{array}$ \\
\hline IMME $\left(\mathrm{kg} / \mathrm{m}^{2}\right)$ & $17,35( \pm 3,13)$ & $\begin{array}{c}19,83 \\
( \pm 3,12)\end{array}$ & $\begin{array}{c}18,9 \\
( \pm 3,31)\end{array}$ \\
\hline Albumina (mg/dL) & $3,72( \pm 0,31)$ & $3,77( \pm 0,33)$ & $\begin{array}{c}3,75 \\
( \pm 0,32)\end{array}$ \\
\hline PCR (mg/dL) & $0,746( \pm 0,56)$ & $0,60 \underline{(+0,76)}$ & $\begin{array}{c}0,65 \\
( \pm 0,69)\end{array}$ \\
\hline Creatinina (mg/dL) & $1,72( \pm 0,6)$ & $2,14( \pm 0,8)$ & $\begin{array}{c}1,98 \\
( \pm 0,76)\end{array}$ \\
\hline $\mathrm{RFGe}(\mathrm{mL} / \mathrm{min})$ & $\begin{array}{c}37,16 \\
(+13,69) \\
\end{array}$ & $\begin{array}{c}36,85 \\
(+12,01) \\
\end{array}$ & $\begin{array}{c}36,97 \\
( \pm 12,46)\end{array}$ \\
\hline
\end{tabular}

$n$ : número absoluto; IMC: índice de massa corporal; CA: circunferência abdominal; $C B(\%)$ : percentual de adequação de circunferência do braço; PCT (\%): percentual de adequação prega cutânea triciptal; CMB (\%): percentual de adequação circunferência muscular do braço; MLG (\%): percentual de massa livre de gordura; GC (\%): percentual gordura corporal; IMME: índice de massa esquelética; PCR: proteína $C$ reativa; RFGe: ritmo de filtração glomerular estimado 
Tabela 2. Parâmetros antropométricos estratificados em risco cardiovascular por meio da circunferência abdominal

\begin{tabular}{|c|c|c|c|c|}
\hline & \multicolumn{3}{|c|}{ Circunferência Abdominal } & \multirow[t]{2}{*}{ p-valor } \\
\hline & Sem risco & Risco & Alto Risco & \\
\hline IMM & 18,13 & 17,65 & 20,23 & 0,203 \\
\hline$E$ & $( \pm 3,3)$ & $( \pm 2,75)$ & $( \pm 3,38)$ & \\
\hline \multirow[t]{2}{*}{ IMC } & 24,26 & 26,28 & 31,53 & 0,0008 \\
\hline & $( \pm 3,4)^{\mathrm{a}}$ & $( \pm 3,2)^{\mathrm{a}}$ & $( \pm 4,76)^{b}$ & \\
\hline \multirow[t]{2}{*}{$\% \mathrm{CB}$} & 90 & 101 & 114,97 & 0,0017 \\
\hline & $( \pm 14,99)^{a}$ & $( \pm 8,84)^{\mathrm{a}}$ & $( \pm 14,03)^{b}$ & \\
\hline$\%$ & 100,5 & 93 & 128,83 & 0,2176 \\
\hline PCT & $(+44,02)$ & $(+41,98)$ & $(+54,42)$ & \\
\hline \multirow[t]{2}{*}{ PCR } & 0,49 & 0,47 & 0,91 & 0,4847 \\
\hline & $( \pm 0,29)$ & $( \pm 0,26)$ & $( \pm 1,02)$ & \\
\hline \multirow[t]{2}{*}{$\% G C$} & 24,85 & 33,22 & 35,43 & 0,0157 \\
\hline & $( \pm 6,76)^{a}$ & $( \pm 3,78)^{b}$ & $( \pm 10,21)^{b}$ & \\
\hline
\end{tabular}

IMME: índice de massa esquelética; IMC: índice de massa corporal; $C B(\%)$ : percentual de adequação de circunferência do braço; PCT (\%): percentual de adequação prega cutânea triciptal; PCR: proteína $C$ reativa; GC: gordura corporal * $p<0,05$

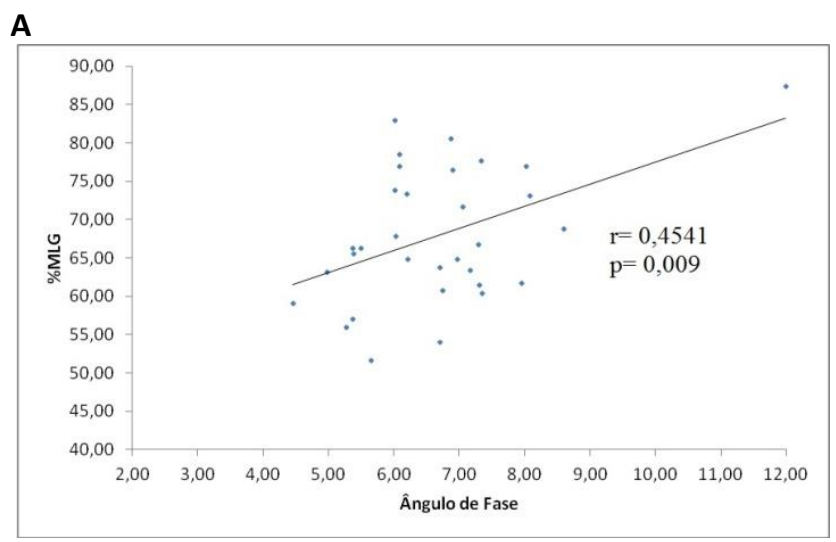

B

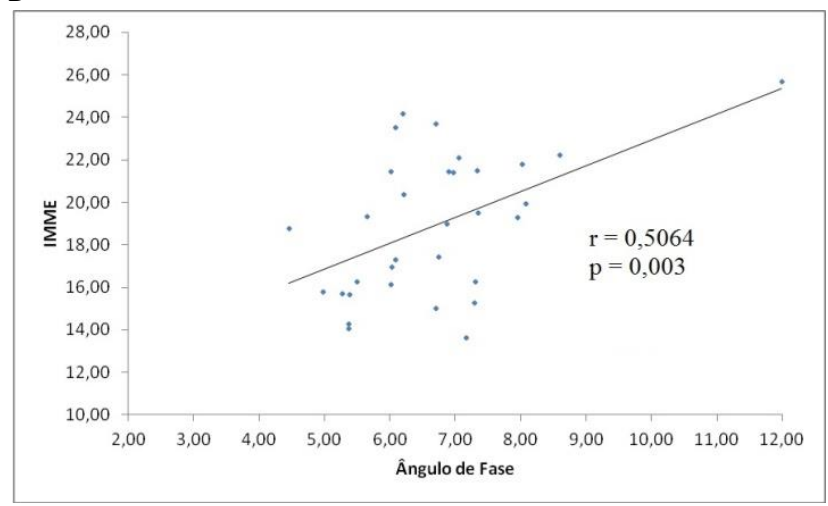

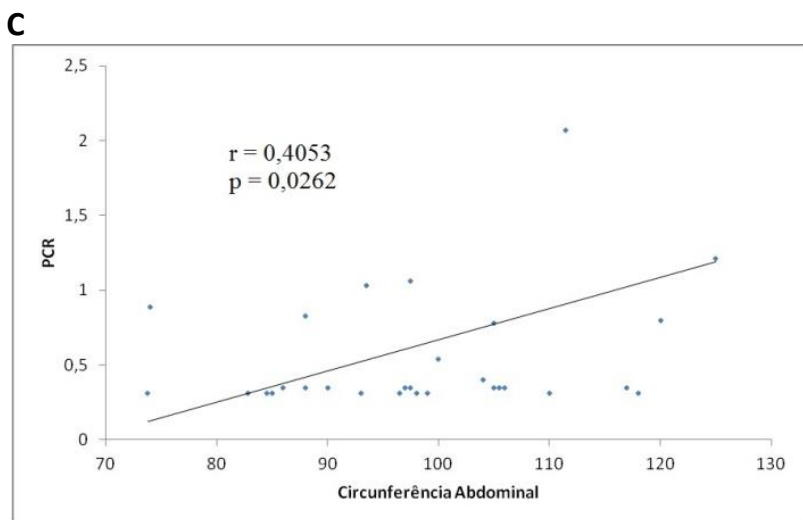

Figura 1. Correlações entre ângulo de fase e percentual de massa livre de gordura (\%MLG) (A), ângulo de fase e índice de massa esquelética (IMME) (B), e entre circunferência abdominal e Proteina C Reativa (PCR) (C).

\section{DISCUSSÃO}

No presente estudo, pode-se observar uma maior prevalência de indivíduos idosos portadores de DRC, fato este que se justifica pela transição demográfica, expressa pelo aumento progressivo da população idosa e a consequente alteração no perfil de morbi-mortalidade, com aumento da incidência de doenças crônicas não-transmissíveis, como a DRC. Concomitantemente, observou-se que a hipertensão arterial e diabetes melittus foram as comorbidades apresentadas pelos pacientes avaliados, as quais estão entre as principais causas da DRC ${ }^{27}$.

Os parâmetros antropométricos revelaram que os doentes estudados estão com sobrepeso/obesidade, indo ao encontro do que acontece mundialmente, o aumento do número de obesos de forma endêmica ${ }^{28}$.Tem-se descrito que a obesidade é um fator de risco independente para o desenvolvimento de diabetes mellitus e hipertensão arterial,. KRAMER e colaboradores $(2006)^{13}$ verificaram o aumento significativo de obesidade em um grupo de pacientes com DRC em fase inicial de hemodiálise de 1995 a 2002.

Concomitantemente ao aumento da obesidade entre os doentes renais crônicos, tem se observado o fenômeno da sarcopenia do obeso, síndrome caracterizada pela perda progressiva e generalizada do tecido muscular esquelético e de força muscular, acarretando em aumento no risco de adversidades como piora na qualidade de vida, desordens fisiológicas e até a morte ${ }^{29}$. Este achado quando em indivíduos obesos, chama-se de sarcopenia do obeso30. Honda et al $(2007)^{14}$ no estudo com 328 pacientes verificou a correlação entre desnutrição pela ASG e a sarcopenia do obeso. A desnutrição foi 
preditor de mortalidade em obesos e não obesos e tanto a desnutrição quanto a obesidade estiveram associadas à inflamação. Pacientes eutróficos pela ASG e com IMC elevado tiveram maior sobrevida. Também foi evidenciada associação de alta \%GC, baixa \%MLG, inflamação e mortalidade.

No presente estudo, pode-se observar que o $A F$ teve relação com o IMME, \%MLG, entretanto não houve relação com as demais medidas antropométricas. Assim, o AF parece identificar alterações de composição corporal que antecedem alterações de parâmetros antropométricos, sendo preditor precoce de desnutrição. De acordo com a literatura, sabe-se que baixos valores de ângulo de fase estão associados à morte celular ou a alguma alteração na permeabilidade seletiva da membrana ${ }^{5,6}$. A desnutrição por sua vez acarreta alterações da integridade da membrana celular ${ }^{5}$. O AF parece estimar valores de massa celular corporal, assim baixos valores de AF podem ser interpretados ainda como desnutrição, definido pela redução da massa celular corporal $^{31,32}$ que pode acontecer mesmo na ausência de modificações de peso e massa magra ${ }^{31}$. Por indicar morte celular, pode ainda ser utilizado como indicador de agravamento da doença, com consequente aumento da morbimortalidade ${ }^{5,6}$

Ao analisar o estado nutricional por outros parâmetros, observou-se que houve associação significativa entre AF e \%MLG e ASG. Estes resultados também foram observados no estudo realizado por OLIVEIRA6, com 58 pacientes em hemodiálise, este estudo demonstrou uma associação entre o $A F$ com ASG e \%MLG. VANNINI et al (2009) ${ }^{33}$, estudaram 52 pacientes hemodialíticos e verificaram que o AF e ASG estiveram associados, o que demonstra a aplicabilidade do SGA enquanto parâmetro de desnutrição em DRC dialíticos.

Os valores da CA estão acima do recomendado nos pacientes avaliados, caracterizando risco cardiovascular. Aqueles com CA com muito risco obtiveram valores de $\% \mathrm{GC}$ superiores, evidenciando que valores de CA refletem o \%GC. Observou-se também uma relação positiva entre CA e PCR, mostrando que a relação inflamatória esta associada ao acúmulo de gordura visceral. No estudo conduzido na cidade de São Paulo com 122 pacientes $^{34}$ não dialíticos, foi comparada a CA com a gordura visceral verificada pela tomografia computadorizada. Os autores concluíram que a CA, por estar fortemente associada à gordura visceral, pode ser utilizada como uma ferramenta de avaliação do risco cardiovascular simples e de baixo custo nesta população.

O IMC médio dos pacientes avaliados está acima do recomendado também, caracterizando risco cardiovascular. No estudo longitudinal de 1 ano, na cidade do Rio de Janeiro ${ }^{34}$, com 44 pacientes nos estagios 3 e 4 , observou-se que a mai oria dos pacientes encontravam-se em sobrepeso/obesidade de acordo com os parâmetros antropométricos avaliados. $O$ estudo sugere que intervenções com o objetivo de reduzir o peso e CA em pacientes predialiticos podem reduzir a resposta inflamatória sistêmica.

A obesidade pode ser identificada por meio de diversos parâmetros, como CA, IMC, \%GC. Entretanto, IMC compreende tanto músculo quanto gordura periférica, enquanto que a CA estima especificamente gordura abdominal, principal como preditor de risco cardiovascular. Os estudos que avaliam obesidade e mortalidade em DRC têm utilizado na maior parte das vezes apenas o IMC. Como pode ser observado no estudo de coorte de KRAMER et al $(2011)^{36}$ com 5805 pacientes com DRC nos estágios 1 a 4, em que avaliou o IMC, em conjunto com CA, pode-se verificar um maior risco de mortalidade associada à obesidade na população estudada.

Similarmente, POSTORINO (2009) $)^{12}$ demonstrou em um estudo de coorte prospectivo, com 537 pacientes com DRC, que o aumento no risco de mortalidade foi relacionado a várias causas, incluindo as cardiovasculares, em pacientes com obesidade abdominal $(p<0,001)$, e IMC abaixo da mediana Estes resultados indicam a necessidade de associar os parâmetros nutricionais para garantir maior precisão nas relações de risco e na prevenção de morbimortalidade dos pacientes.

Observou-se no presente estudo que não houve diferença entre os grupos com distintas classificações de CA em relação ao IMME, o que indica que a gordura visceral e massa muscular não apresentam relação, ou seja, o fato de apresentar maior gordura visceral não significa que o individuo possua menos massa muscular. Estes resultados sugerem outros estudos para explorar a composição corporal utilizando o método padrão ouro como o DEXA (Dual-emission X-ray absorptiometry).

\section{CONCLUSÃO}

Nesta casuística, apesar da pequena amostragem de pacientes, os indivíduos portadores de DRC em tratamento conservador apresentaram dados antropométricos que indicam sobrepeso, obesidade e acúmulo de gordura corporal. O AF parece identificar alterações de composição corporal que antecedem alterações de parâmetros antropométricos. Pode-se verificar que a ASG pode ser uma ferramenta adequada na avaliação nutricional desta população e que em nosso meio a abordagem nutricional deve ser focalizada a fim de diminuir o risco cardiovascular que e potencializado pela presença de DRC. 


\section{REVISTA MÉDICA DA UFPR}

\section{CONFLITOS DE INTERESSE}

Não houve conflito de interesses.

\section{REFERÊNCIAS}

1. Leite IdaC, Schramm JMdeA, Gadelha AMJ, Valente JG, Campos MR, Portela MC, et al . Comparação das informações sobre as prevalências de doenças crônicas obtidas pelo suplemento saúde da PNAD/98 e as estimadas pelo estudo Carga de Doença no Brasil. Ciênc. saúde coletiva. 2002; 7(4): 733-741.

2. Lessa I. Níveis séricos de creatinina: hipercreatininemia em segmento da população adulta de Salvador, Brasil. Rev. bras. epidemiol. [serial on the Internet]. 2004; 7(2): 176-186.

3. Nihi MM, Manfro RC, Martins C, Suliman M, Murayama $Y$, Riella $\mathrm{MC}$, et al. Associação entre gordura corporal, inflamação e estresse oxidativo na hemodiálise. J. Bras. Nefrol. 2010;32(1):11-17.

4. Bellizzi V, Scalfi L, Terracciano V, De Nicola L, Minutolo R, Marra M, Guida B, et al. Early changes in bioelectrical estimates of body composition in chronic kidney disease. J Am Soc Nephrol. 2006 May;17(5):1481-7.

5. Gupta D, Lammersfeld CA, Burrows JL, Dahlk SL, Vashi PG, Grutsch $\mathrm{JF}$, et al. Bioelectrical impedance phase angle in clinical practice: implications for prognosis in advanced colorectal cancer. Am J Clin Nutr. 2004 Dec;80(6):1634-8.

6. Oliveira CM, Kubrusly M, Mota RS, Silva CA, Choukroun G, Oliveira VN. The phase angle and mass body cell as markers of nutritional status in hemodialysis patients. J Ren Nutr. 2010; 20(5):314-20.

7. Jha V, Jairam A, Sharma MC, Sakhuja V, Piccoli A, Parthasarathy S. Body composition analysis with bioelectric impedance in adult Indians with ESRD: comparison with healthy population. Kidney Int. 2006;69(9):1649-53.

8. Elckemberg $M$, Oliveira CCde; Roriz AKC, Sampaio LR. Bioimpedância elétrica e sua aplicação em avaliação nutricional. Rev. Nutr. [serial on the Internet]. 2011 Dec [cited 2012 Mar 29] ; 24(6): 873-882.

9. Lara CB Pupim, Cacilda B. Ribeiro, Pamela Kent e Talat A. Ikizler. Atualização em diálise: uso da impedância bioelétrica em pacientes em diálise. J. Bras. Nefrol. 2000;22(4):249-256.

10. Nagano M, Suita S, Yamanouchi T. The validity of bioelectrical impedance phase angle for nutritional assessment in children. J Pediatr Surg. 2000;35(7):1035-9.

11. Toso S, Piccoli A, Gusella M, Menon D, Bononi A, Crepaldi G, et al. Altered tissue electric properties in lung cancer patients as detected by bioelectric impedance vector analysis. Nutrition. 2000;16(2):1204

12. Postorino M, Marino C, Tripepi G, Zoccali C; CREDIT (Calabria Registry of Dialysis and Transplantation) Working Group. Abdominal obesity and all-cause and cardiovascular mortality in end-stage renal disease. J Am Coll Cardiol. 2009; 53(15):1265-72.

13. Kramer HJ, Saranathan A, Luke A, Durazo-Arvizu RA, Guichan C, Hou $S$, et al. Increasing body mass index and obesity in the incident ESRD population. J Am Soc Nephrol. 2006;17(5):1453-9.
14. Honda H, Qureshi AR, Axelsson J, Heimburger O, Suliman ME, Barany $P$, et al. Obese sarcopenia in patients with end-stage renal disease is associated with inflammation and increased mortality. Am J Clin Nutr. 2007; 86(3):633-8.

15. National Kidney Foundation. K/DOQI Clinical Practice Guidelines for Chronic Kidney Disease: Evaluation, Classification and Stratification. Am J Kidney Dis 39:S1-S266, 2002 (suppl 1)

16. Detsky AS, Mclaughlin JR, Baker JP, Johnston N, Whittaker S, Mendelson RA, et al. What is Subjective Global Assessment of Nutritional Status? Journal JPEN J Parenter Enteral Nutr. 1987;11(1):8-13.

17. Detsky AS, Smalley PS, Chang J. What Is This Patient Malnourished? JAMA. 1994;271(1):54-58.

18. WHO - WORLD HEALTH ORGANIZATION. Obesity: preventing and managing the global epidemic. Report of the WHO Consultation on Obesity. Division of Noncommunicable Diseases, Programme of Nutrition Family and Reproductive Health, Geneva, 1998.

19. Lipschitz DA. Screening for nutritional status in the elderly. Prim Care. 1994;21(1):55-67.

20. Frisancho AR. New norms of upper limb fat and muscle areas for assessment of nutritional status. Am J Clin Nutr. 1981; 34(11):2540 2545.

21. Frisancho, A. R. Anthropometric Standards for the Assessment of Growth and Nutrition Status. The University of Michigan Press, Michigan, p. 189, 1990.

22. Burr ML, Phillips KM. Anthropometric norms in the elderly. Br J Nutr. 1984;51:165-9.

23. Blackburn GL, Thornton PA. Nutritional assessment of the hospitalized patient. Med Clin North Am. 1979;63(5):11103-15.

24. Associação Brasileira para o Estudo da Obesidade e da Síndrome Metabólica. Diretrizes brasileiras de obesidade 2009/2010 / ABESO Associação Brasileira para o Estudo da Obesidade e da Síndrome. Metabólica. - 3.ed. - Itapevi, SP : AC Farmacêutica, 2009.

25. Baumgartner RN, Chumlea WC, Roche AF. Bioelectric impedance phase angle and body composition. Am J Clin Nutr. 1988;48(1):16-23.

26. Janssen I, Baumgartner RN, Ross R, Rosenberg IH, Roubenoff R. Skeletal Muscle Cutpoints. Associated with Elevated Physical Disability Risk in Older Men and Women. Am J Epidemiol. 2004; 159: 413-21.

27. Kusumota L, Rodrigues RAP, Marques S. Idosos com insuficiência renal crônica: alterações do estado de saúde. Rev. Latino-Am. Enfermagem. 2004; 12(3): 525-532.

28. Navaneethan SD, Yehnert H, Moustarah F, Schreiber MJ, Schauer PR, Beddhu S. Weight loss interventions in chronic kidney disease: a systematic review and meta-analysis. Clin J Am Soc Nephrol. 2009 Oct;4(10):1565-74

29. Cruz-Jentoft AJ, Baeyens JP, Bauer JM, Boirie Y, Cederholm T, Landi $F$, et al. Sarcopenia: European consensus on definition and diagnosis. Report of the European Working Group on Sarcopenia in Older People. Age and Ageing 2010; 39: 412-423.

30. Stenholm S, Harris TB, Rantanen T, Visser M, Kritchevsky SB, Ferrucci L. Sarcopenic obesity - definition, etiology and consequences. Curr Opin Clin Nutr Metab Care. 2008 November; 11(6): 693-700. 


\section{REVISTA MÉDICA DA UFPR}

31. Johansen KL, Kaysen GA, Young BS, Hung AM, Silva Mda, Chertow GM. Longitudinal study of nutritional status, body composition, and physical function in hemodialysis patients. Am J Clin Nutr. 2003;77:842-6.

32. Barbosa-Silva MCG, Barros AJD. Bioelectrical impedance analysis in clinical practice: a new perspective on its use beyond body composition equations. Curr Opin Clin Nutr Metab Care. 2005; 8:311-317.

33. Vannini FD, Antunes AA, Caramori JC, Martin LC, Barretti P. Associations between nutritional markers and inflammation in hemodialysis patients. Int Urol Nephrol. 2009; 41(4):1003-9.

34. Sanches FM, Avesani CM, Kamimura MA, Lemos MM, Axelsson J, Vasselai $P$, Draibe $S A$, et al. Waist circumference and visceral fat in CKD: a cross-sectional study. Am J Kidney Dis. 2008; 52(1):66-73.

35. Carvalho LK, Barreto Silva MI, da Silva Vale B, Bregman R, Martucci RB, Carrero JJ, et al. Annual variation in body fat is associated with systemic inflammation in chronic kidney disease patients Stages 3 and 4: a longitudinal study. Nephrol Dial Transplant. 2011 Aug 8.

36.Kramer H, Shoham D, McClure LA, Durazo-Arvizu R, Howard G, Judd $S$, et al.Association of waist circumference and body mass index with allcause mortalitl in CKD: The REGARDS (Reasons for Geographic and Racial Differences in Stroke) Study. Am J Kidney Dis. 2011; 58(2):177-85. 